\title{
On a Method of Preventing the Ground-Reflected Wave Effect in Rawin Observation
}

\author{
by \\ K. Naito \\ Meteorological Research Institute \\ (Received December 21, 1950)
}

\begin{abstract}
In rawin observation, we face many troubles, one of which is caused by ground-reflected waves. A conductive screen of knife-edge type is presented to get rid of this trouble with verified effectiveness. The method to determine the screen position and height is given based on the diffraction theory.
\end{abstract}

\section{Introduction}

In rawin observations, the ground-reflected waves deteriorate the accuracy of elevation angle measurement. Especially, the lower part of elevation angles is observed with considerable errors. (1)

As one method of avoiding the detrimental influence of the ground-reflected waves, a conductive plane screen of knife-edge type may be placed in front of the receiving antenna. The height and position of the screen should be determined so that the screen would block the reflected waves with least disturbance to the direct ones.

Many diffraction theories of electromagnetic waves have been propounded until now, none of which, however, has been corroborated to the minute points in actual applications. In optics, however, these theories can well explain the light diffraction phenomena, and they are now admitted to be applied to the microwave phenomena, too. (2);(3) Therein, we intend to use the same idea for our purfose.

\section{Diffraction of plane electromagnetic waves by a conductive plane screen}

We proceed according to the method usually adopted in optics. (4)

In a homogeneous and isotropic medium, let $E$ and $e^{i \omega t}$ derote the electric field and its time factor respectively.

$$
\left\{\begin{array}{l}
E=u(x, y, z) e^{i \omega t}, \\
\Delta u+k^{2} u=0,
\end{array} \quad(k=2 \pi / \lambda),\right.
$$

where $\lambda$ is the wave length. Under the assumption of some regularities, the equation (1) may be written according to Kirchhoff in the form

$$
U(P)=-\frac{1}{4 \pi} \iint_{S}\left\{u \frac{\partial}{\partial \nu}\left(\frac{e^{-i k r}}{r}\right)-\frac{e^{-i k r}}{r} \frac{\partial u}{\partial \nu}\right\} d a,
$$

where $P$ is in a closed surface $S$ with outward normal $\nu$ and $r$ is the distance from a point on $S$ to $P$. 
Consider now a plane wave incident upon a completely conductive plane screen with an aperture. Take a Cartesian coordinate system in the plane of the screen, the origin being chosen at a point in the plane where the wave normal through $P$ pierces the screen. Let the $x$-axis coincide with the projection of the wave ray on the screen and the positive $z$-axis be directed toward $P$, that is, opposite to the wave source. In this case, the diffracted wave is expressed from (2) by

$$
U(P)=\frac{i k E_{0}}{2 \pi} \cos \delta \frac{e^{-k\left(n \cdot r_{0}+R\right)}}{R} \times \iint e^{-\frac{i k}{R}\left(\xi^{2} \cos ^{2} \delta+\eta^{2}\right)} d \xi d \eta,
$$

where $E_{0} e^{-k(n \cdot r)+i \omega t}$ is the incident plane wave,

$n$, the unit propagation vector of the plane wave,

$\delta$, the angle between the screen and the wave front,

$-k n \cdot r_{0}$. the phase of the wave at the origin,

$R$, the distance from the origin to $P$ and

$\xi$, $\eta$ the coordinates of an elementary area of the aperture in the screen.

Moreover it is assumed that $R$ is sufficiently large and $1 / R$ may be neglected compared with $K$.

In calculating the diffraction by the knife-edge type screen, the integration is carried out over the domain defined by

(4)

$$
\left\{\begin{array}{r}
x_{0}<\xi<\infty, \\
-\infty<n<\infty,
\end{array}\right.
$$

where $x_{0}$ denotes the distance from the top of the screen to the origin. Using Fresnel's integral

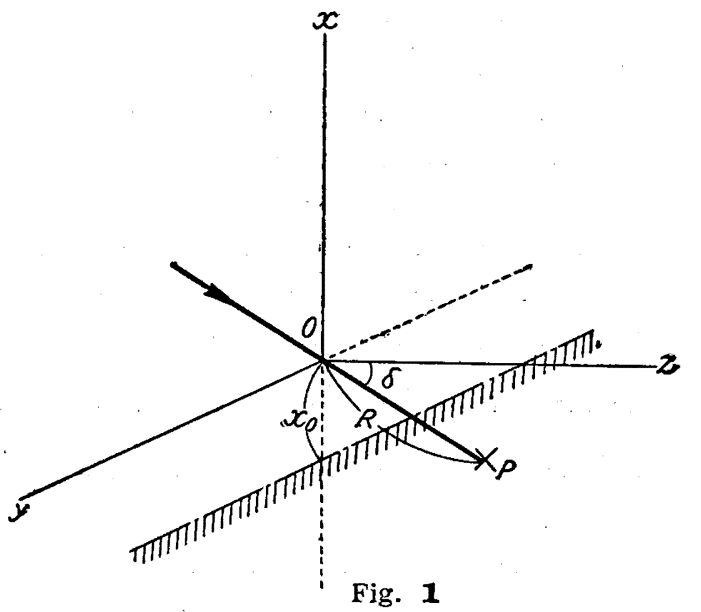

$$
\begin{aligned}
& C\left(w_{0}\right)=\int_{0}^{w_{0}} \cos \left(\frac{\pi}{2} u^{2}\right) d u, \\
& S\left(w_{0}\right)=\int_{0}^{w_{0}} \sin \left(\frac{\pi}{2} u^{2}\right) d u,
\end{aligned}
$$

the equation (3) is transformed into the following form:

(5)

$$
\left\{\begin{aligned}
U(P) & =E_{0} e^{-i k\left(n \cdot r_{0}+R\right) \vartheta e^{i} \psi,} \\
\vartheta & =\frac{1}{\sqrt{2}} \sqrt{\left(\frac{1}{2} \pm C\left(w_{0}\right)\right)^{2}+\left(\frac{1}{2} \pm S\left(w_{0}\right)\right)^{2}}, \\
\psi & =\frac{\left\{\left(\frac{1}{2} \pm C\left(w_{0}\right)\right)-\left(\frac{1}{2} \pm S\left(w_{0}\right)\right)\right\}}{\left\{\left(\frac{1}{2} \pm C\left(w_{0}\right)\right)+\left(\frac{1}{2} \pm S\left(w_{0}\right)\right)\right\},} \\
w_{0} & =\left|x_{0}\right| \cos \delta \sqrt{\frac{2}{\lambda R}} .
\end{aligned}\right.
$$

((Upper and lower signs of \pm correspond to $x_{0}<0$ and $x_{0}>0$ respectively.) 
As $E_{0} e^{-i k\left(n \cdot r_{0}+R\right)}$ is the wave at $P$ without the screen, the effect of the screen is shown by $\vartheta e^{i} \psi$. Now we note that the equation (2), i. e., the expression for a definite aperture, is not of suitable form for the integration domain (4). As may be seen later, it is the upper limit of the magnitude of screen effect that we really need. So we shall use temporarily the equation (2) as an approximation.

Next we proceed to the case shown in Fig. 2, in which a conductive plane screen of knife-edge type is placed on an even ground, and a plane wave, with the incident angle $\left(\frac{\pi}{2}-\delta\right)$ against the ground propagates in a plane normal to the screen.

The special cases for the microwave may be treated approximately in the following manner. In Fig. 2, $\boldsymbol{E}_{0}$ is taken as a plane wave for the $A$-direction (including phase relation)

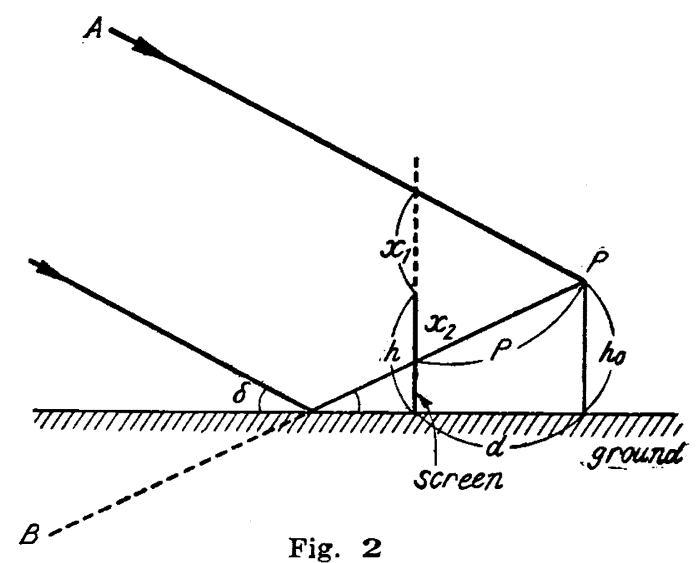
and then the reflected wave is a plane wave from the B-direction with the reflection coefficient $R e^{i \phi}$ on the ground. The field at $P$ therefore turns out to be the superposition of the $A$-wave and the $B$-wave, both diffracted by the screen. As we treat only a plane wave, the reflection coefficient $R e^{i \phi}$ may well be determined for the incident angle $\left(\frac{\pi}{2}-\delta\right)$, which is found to be more complicated if we consider a non-plane wave case and the meaning shown by the integration in (3) is taken into account.

As for the reflection coefficient, we may use the expressions for the case in which one medium is purely dielectric with $(\varepsilon, \mu)$ and the other is conductive with $\left(\sigma_{e}\right.$, $\left.\varepsilon_{e}, \mu_{e}\right)$. ${ }^{(5)}$ Generally speaking, the reflection coefficient depends on the polarization of waves. For example, if we regard $\rho_{1} e^{i \phi 1}$ as the reflection coefficient of the polarized electric vector normal to the incident plane and $\rho_{\mu 2} e^{i \phi 11}$ as that of the polarized one parallel to the incident plane,

$$
\begin{aligned}
& \rho_{1}^{2}=\frac{\left(\mu q-\mu_{e} \alpha \cos \theta_{0}\right)^{2}+\mu^{2} p^{2}}{\left(\mu q+\mu_{e} \alpha \cos \theta_{0}\right)^{2}+\mu^{2} p^{2}} \\
& \tan \rho_{1} e^{i \phi_{I}}=\frac{2 \mu_{e} \mu \alpha p \cos \theta_{0}}{\mu^{2}\left(q^{2}+p^{2}\right)-\mu_{e}^{2} \alpha^{2} \cos ^{2} \theta_{0}}, \\
& \rho^{2}{ }_{11}=\frac{\left[\mu\left(\alpha_{e}{ }^{2}-\beta_{e}{ }^{2}\right) \cos \theta_{0}-\mu_{e} \alpha q\right]^{2}+\left[2 \mu \alpha_{e} \beta_{e} \cos \theta_{0}-\mu_{e} \alpha p\right]^{2}}{\left[\mu\left(\alpha_{e}{ }^{2}-\beta_{e}{ }^{2}\right) \cos \theta_{0}+\mu_{e} \alpha q\right]^{2}+\left[2 \mu \alpha_{e} \beta_{0} \cos \theta_{0}+\mu_{e} \alpha p\right]^{2}}, \\
& \tan \phi_{1 l}=\frac{2 \mu_{e} \mu \alpha p\left(q^{2}+p^{2}-\alpha^{2} \sin ^{2} \theta_{0}\right) \cos \theta^{0}}{\mu^{2}\left(\alpha_{e}{ }^{2}+\beta_{e}{ }^{2}\right) \cos ^{2} \theta_{0}-\mu_{e}^{2} \alpha^{2}\left(q^{2}+p^{2}\right)^{\prime}}, \\
& k_{a}^{2}=\omega^{2} \varepsilon_{0} \mu_{0}+i \omega \sigma_{0} \mu_{0}=\left(\alpha_{0}+i \beta_{0}\right)^{2} \text {, } \\
& k=\omega \sqrt{\varepsilon \mu}=\alpha \text {, }
\end{aligned}
$$




$$
\begin{aligned}
& p^{2}=\frac{1}{2}\left[-\alpha_{e}{ }^{2}+\beta_{e}{ }^{2}+a^{2} \sin ^{2} \theta_{0}+\sqrt{4 a_{e}^{2} \beta_{e}{ }^{2}+\left(\alpha_{e}{ }^{2}-\beta_{e}{ }^{2}-a^{2} \sin ^{2} \theta_{0}\right)^{2}}\right], \\
& q^{2}=\frac{1}{2}\left[\alpha_{e}^{2}-\beta_{e}{ }^{2}-a^{2} \sin ^{2} \theta_{0}+\sqrt{4 a_{e}{ }^{2} \beta_{e}{ }^{2}+\left(a^{2}-\beta_{e}{ }^{2}-a^{2} \sin ^{2} \theta_{0}\right)^{2}}\right],
\end{aligned}
$$

where $\theta_{0}$ is the incident angle. If the polarization is more general than the above, the reflection coefficient is naturally more complicated. If the dielectric medium is the air and the conductive medium the earth (earth's specific dielectric capacity and conductivity are about 6 and $10^{-10}$ in rational M.K.S. units respectively), König's approximation for the reflection coefficient may be used at frequencies more than $1 \mathrm{Mc} / \mathrm{s}$, as proved experimentally by Pfannenberg ${ }^{(7)}$ and is shown in Fig. 3.

3. Determination of the position and height of the screen

According to (5), the effect of the screen depends solely on $w_{0}$. For our purpose of preventing the ground-reflected waves, it can simply be stated that $x_{0}$ should be negative for direct waves and positive for reflected waves; in other words, $P$ should be in the geometrical shadow of reflected waves. If $w_{0 i}$ for direct waves and $w_{0 r}$ for reflected waves are suitable ones as $w_{0}$ respectively, from the equation of (5) we get, by referring to Fig. 2 ,

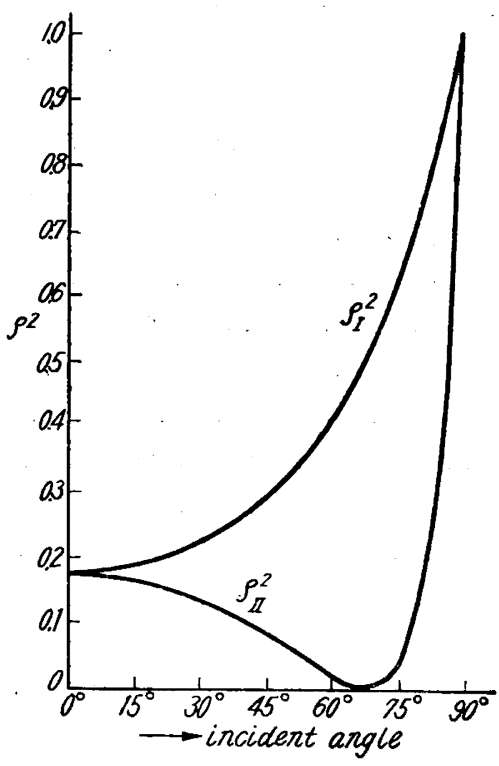

Fig. 3

$$
\left\{\begin{aligned}
w_{0 i} & =\cos \delta \sqrt{\frac{2}{\lambda}} \frac{x_{1}}{\sqrt{R}} \\
w_{0 r} & =\cos \delta \sqrt{\frac{2}{\lambda}} \frac{x_{2}}{\sqrt{R}} \\
x_{1}+x_{2} & =2 R \sin \delta
\end{aligned}\right.
$$

Therefore, we obtain

$$
\begin{gathered}
R=\frac{\lambda\left(w_{0 i}+w_{0 r}\right)^{2}}{2 \sin ^{2}(2 \delta)}, \\
x_{1}=\frac{\lambda w_{0 \delta}\left(w_{0 i}+w_{0 r}\right)^{2}}{4 \cos ^{2} \delta \sin \delta} .
\end{gathered}
$$

And by simple geometrical calculations we have

$$
\left\{\begin{array}{l}
d=\frac{\lambda\left(w_{01}+w_{0 r}\right)^{2} \cos \delta}{2 \sin ^{2}(2 \delta)}, \\
h=h_{0}+\frac{\lambda\left(w_{08}{ }^{2}-w_{0 r}{ }^{2}\right)}{8 \cos ^{2} \delta \sin \delta},
\end{array}\right.
$$

which are the equations to determine the position and height of the screen. But 
it should be noted that $d$ and $h$ in (8) are unsuitable in some actual cases, because we utilized an equivalent plane wave instead of the existence of the ground. In these cases we must try another suitable $w_{0}$. It is difficult to express such cases mathematically, but one example may be led from the condition $h>0$ and shown by

$$
w_{0 i}^{2}-w_{0 r}^{2}<\frac{8 h_{0}}{\lambda} \cos ^{2} \delta \sin \delta .
$$

In the choice of the values of $w_{0 i}$ and $w_{0 r}$, we must consider the directivity and other characteristics of the receiving antenna and this procedure is rather laborious when the latters are not linear.

If we determine the values of $w_{0 i}$ and $w_{0 r}$ for one value of $\delta$, the position and height of the screen will be consequently calculated. By considering (5), however, it may be difficult to conclude generally that this screen is optimum also for other values of $\delta$. In rawin observations, the prevention of ground-reflected waves is fortunately demanded at lower elevation angles. As the elevation angle approaches to 0 , one-point observation has obviously a lot of errors even without the effect of the reflected waves, and so measurements of very low elevation angles are meaningless. For this reason the band of $\delta$ for us to deal with is necessarily quite narrow. If we are willing to take the trouble, a suitable screen may easily be determined for each $\delta$ in the band and have only to choose the most common and suitable of them.

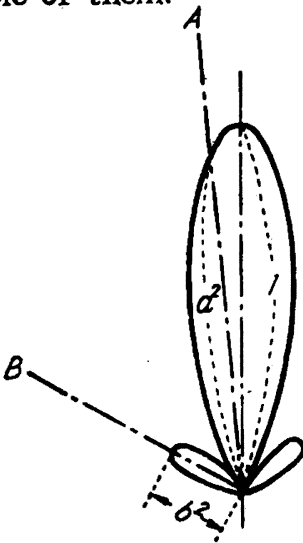

Fig. 4

Next we consider the case in which spurious waves in reception are present, the receiver characteristics being disregarded for the present. Let the directivity of the receiving antenna be shown in Fig. $4, E_{A}$ be a wave from $A$-direction (including phase), and $E_{B} e^{i \psi B}$ be one from $B$-direction, where $\psi_{B}$ is the phase lag to $E_{A}$.

Obviously, the input power $P_{i n}$ is shown by

(10) $P_{i \cdot s}=a^{2} E_{A}^{2}+b^{2} E_{B}{ }^{2}+2 a b E_{A} E_{B} \cos \psi_{B}$.

If A-direction is to be measured and $E_{A}{ }^{2}<P_{i, n}$, a rawin gives wrong direction-finding. If the main lobe of the directivity be considerably sharp, the spurious waves that give wrong direction-finding may be supposed to enter into the side lobes. Putting $a^{2}=1, b^{2}=$ maximum of side lobes and $\cos \psi_{B}=1$ in (10), we have

$$
\left(P_{i n}-E_{A}^{2}\right)_{\max }=b^{2} E_{B}^{2}+2 b E_{A} E_{B}
$$

Here we take the diffracted wave by the above-mentioned screen (diftraction coefficient $\vartheta \mathrm{e}^{i} \psi$ ) of the ground- reflected wave (reflection coefficient $\Re \mathrm{e}^{i \phi}$ ) to be $E_{B}{ }^{i \psi_{B}}$, assuming the receiving characteristics to be linear. We can thus obtain the follo wng expression for (11), which will be the criterion in the determination of $w_{0 r}$,

$$
b^{2} \Re^{2} \vartheta^{2} E_{0}{ }^{2}+2 b \Re \vartheta E_{0}{ }^{2} \text {. }
$$

Putting $\left|E_{0}\right|=1$ in this equation, it is expressed as follows : 


$$
b^{2} \Re^{2} \vartheta^{2}+2 b \Re \vartheta
$$

So far, we have considered only diffracted waves of a direct wave and a groundreflected wave as the contributions to the field at $P$. In reality, however, there are also ground-reflected waves of these diffracted waves, which may be regarded negligibly small, judged from the magnitudes of the reflection and diffraction coefficient, and the directivity of the receiving antenna.

\section{Ixamples}

Let $\vartheta_{1}$ be the magnitude of the diffraction coefficient in the case in which $P$ does not lie in the geometrical shadow of the screen, and $\vartheta_{2}$ be the magnitude of the diffraction coefficient in the (8) case in which it does. Then $\vartheta_{1}$ and $\vartheta_{2}$ are shown in Fig. 5 and Fig. 6 respectively.

The method described in Section 3 is so laborious that we shall rather take a rough "cut and try" method to show an example.

Assume that $\delta$ $=15^{\circ}, \quad w_{0 i}=1.2$ and $w_{0 r}=1.7$. If wave length is $73 \mathrm{~cm}$ and height $h_{0}$ of a receiving antenna is $220 \mathrm{~cm}$, from (8) we obtain

$$
\begin{aligned}
& d=1185 \mathrm{~cm}, \\
& h=274.6 \mathrm{~cm} .
\end{aligned}
$$

If we replace $x_{1}$, $x_{2}$ and $R$ by $d$ and $l$ $\left(\equiv h-h_{0}\right)$ in (7) referring to Fig. 2, we get
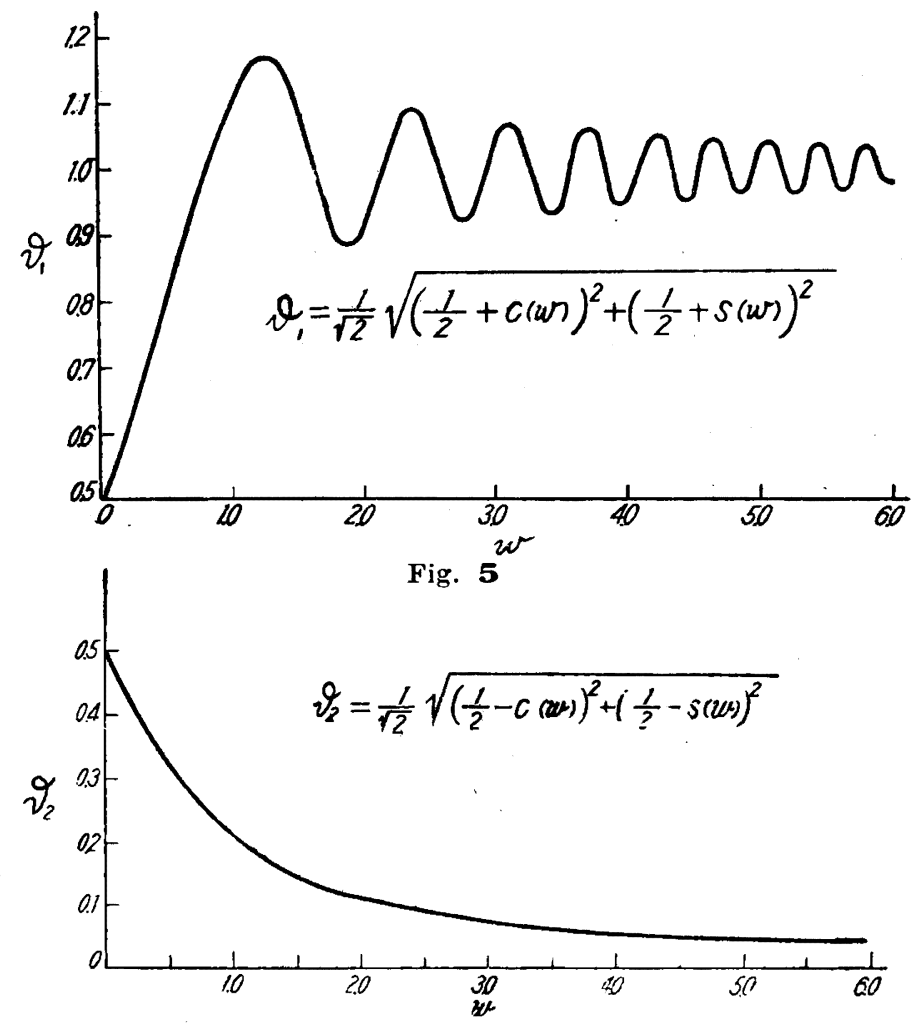

Fig. 6

$$
\begin{aligned}
& w_{0 i}=\sqrt{\frac{2}{\lambda d}} \cos ^{-\frac{3}{2}} \delta(d \tan \delta-l), \\
& w_{0 r}=\sqrt{\frac{2}{\lambda d}} \cos ^{\frac{3}{2}} \delta(d \tan \delta+l) .
\end{aligned}
$$

From these equations, it is easily found that $w_{0 i}$ and $w_{0 r}$ change continuously with $\delta$, and that for $d$ and $h$ obtained above, $d w_{0 i(r)} / d \delta>0$, resulting that the screen is more effective at $\delta=20^{\circ}$ than at $\delta=15^{\circ}$. And numerical calculations give $w_{0 i}=1.64$ and $w_{0 r}=2.12$ at $\delta=20^{\circ}$ showing higher effectiveness. In this case, the magnitude of the diffraction coefficient of the ground-reflected wave is 0.1 as shown in Fig. 6 . 
Reflection coefficient on the ground has various values according to the polarizations of waves as in Fig. 3. As the sending antenna of a rawin does not remain vertical and is continually swayed by wind, the actual state is thought to be intermediate between $\rho_{1}$ and $\rho_{11}$. Hence, we take $\mathfrak{R}^{2}=0.27$. As for $b^{2}$, it is nearly 0.42 in the present rawin. Calculating (12) with these values we get the value of 0.067 , that is to say, the effect of the ground-reflected waves is reduced to several percents in this case. This coincides closely with the

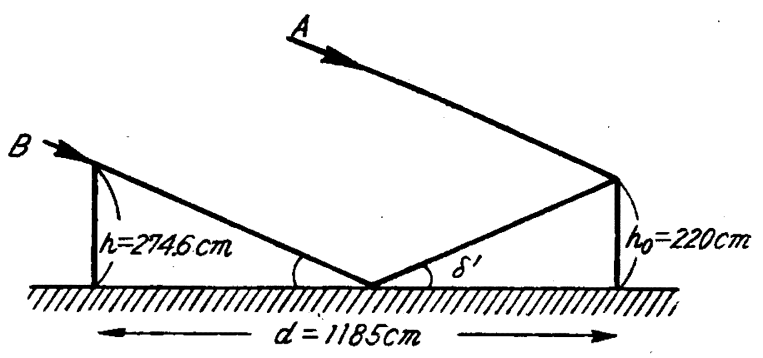

Fig. 7 exactitude of the present receiving apparatus itself. *

The configuration of the screen determined above and the receiving antenna is shown in Fig. 7. From this figure, $\delta^{\prime}$ equals $23^{\circ}$. But this elevation angle $\delta^{\prime}$ does not exist in the region of $\delta$ that concerns us most in rawin observation, because the present rawin is affected by the ground-reflected waves at lower elevation angles than this $\delta^{\prime}$. These discussions indicate that the screen of knife-edge type mentionep above is effective thus far in solving the troubles of the ground-reflected waves for the present rawin. If we follow faithfully the method described in Section 3, we shall of course be able to obtain a more effective one than that obtained above.

We have so far treated of a single screen only, but two or more screens are thought to be more effective in the actual case. As for the phase, it does not matter so long as we use the criterion (12).

\section{Conclusion}

It is shown that the effect of the ground-reflected waves on rawin observation can be prevented by a screen of knife-edge type installed on the ground, and the method is given to determine the optimum screen position and height. It is naturally most important in the application of the method, as in all other actual cases, to treat the problem from the technological stand-point.

At the end of last year the author heard of the success of the above obtained screen in the experiments at Tateno Aerological Observatory, although not detailed.

The author wishes to express his gratitude to Messrs. M Sanuki and N. Kodaira for their kind advices.

\section{APPENDIX}

\section{On a Few Methods of Prevention of the Effect of Reflecting Bodies upon Rawin Observation}

\footnotetext{
* If we use a Braun tube $(120 \mathrm{~mm} \phi)$ as an indicator of the receiver and if the image on the screen of the tube has the linear dimension of about $60 \mathrm{~mm}$, total errors inay be represented as the inage on the screen of linear dimension of several millimeters, taking account of the distinctness of the innage and various errors of all parts of the receiver. This fact tells that the exactitude of the receiving apparatus is the order of several percents.
} 
To prevent the effect of reflecting bodies on rawin observation, sharper directivity in the receiving antenna will be the shortest cut. At present, however, we have no antenna having better directivity than Yagi-antenna now used for the wave length of our rawin. Several Yagi-antenna may be used in the arrangement as a bean antenna, but its actual construction would be a difficult problem fiom the technical point of view.

Therefore, we shall propose some methods apart from the consideration of antenna directivity in the following.

1. For elevation angle

It was mentioned in a previous section that it is desirable to install more than one screen on the ground to make the screening effect complete and the arrangement

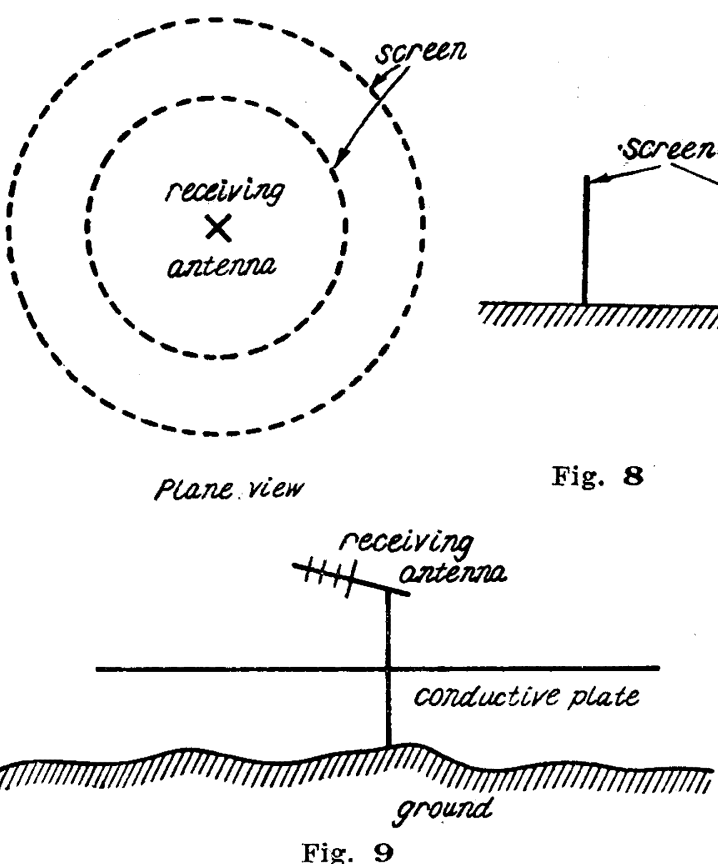

Fig. 9

flected waves with known reflection coefficients ( $c f$. Fig. 9).

This method enables us also to avoid the waves scattered by the uneven ground. In this case we obviously cannot obtain the general expressions of correction terms for reflected waves.

2. For azimuth

In our rawin, the main lobe is so sharp that most of the errors in direction-finding are caused by the side lobes, Hence, we have only to eliminate the side lobes and, for that purpose, two conductive plates like goggles for wagon horses as shown in

of the screens and receiving antenna shown in Fig. 8.

Another method is to place a conductive plane-plate parallel to the ground under the receiving antenna and to make observations always under the influence of re-
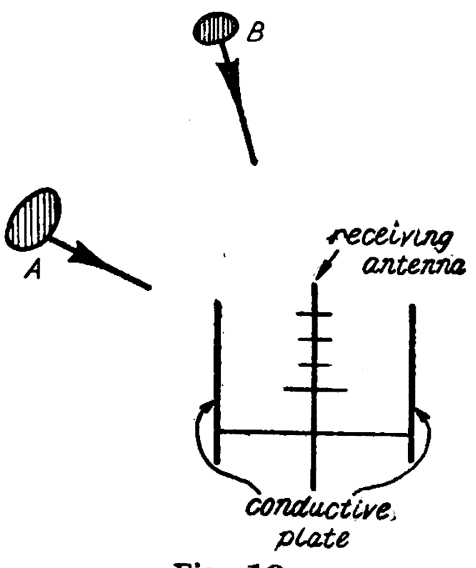

Fig. 10 
Fig. 10 may be used. The arrangement would be valid for an $A$-reflecting body, but not for a B-reflecting body, which probably causes only minor errors.

It goes without saying that we can substitute a metallic wire net of moderate mesh width for the above-mentioned conductive plate. The mesh width should be determined by experiments at present, for existing theories may fail for the purpose.

\section{References}

1. Sakai and Maeda: Kenkyüjiho, Vol. 1, No. 10, 332.

2. For instance, Stratton: Electromagnetic Theory.

3. Stratton and Chu: Phys. Rev. 56, p. 105 ff. 1930.

4. For instance; G. Joos: Theoretical Physics.

5. For instance, Stratton: Electromagnetic Theory.

6. Kornig; Handbuch der Physik, Vol. XX, p. 197 ff.

7. Pfannenberg: Z. Physik, 37, 758, 1926.

8. Jahnke und Einde: Funktionentafeln. 